\title{
QUEEN'S
UNIVERSITY
BELFAST
}

\section{A pharmacist-led medicines review intervention in community-dwelling Mâori older adults- a feasibility study protocol}

Hikaka, J., Hughes, C., Jones, R., Connolly, M. J., \& Martini, N. (2019). A pharmacist-led medicines review intervention in community-dwelling Māori older adults- a feasibility study protocol. Research in Social and Administrative Pharmacy, 16(9), 1264-1271. https://doi.org/10.1016/j.sapharm.2019.12.004

Published in:

Research in Social and Administrative Pharmacy

Document Version:

Peer reviewed version

Queen's University Belfast - Research Portal:

Link to publication record in Queen's University Belfast Research Portal

Publisher rights

(C) 2019 Elsevier Ltd.

This manuscript version is made available under the CC-BY-NC-ND 4.0 license http://creativecommons.org/licenses/by-nc-nd/4.0/,which permits distribution and reproduction for non-commercial purposes, provided the author and source are cited.

\section{General rights}

Copyright for the publications made accessible via the Queen's University Belfast Research Portal is retained by the author(s) and / or other copyright owners and it is a condition of accessing these publications that users recognise and abide by the legal requirements associated with these rights.

Take down policy

The Research Portal is Queen's institutional repository that provides access to Queen's research output. Every effort has been made to ensure that content in the Research Portal does not infringe any person's rights, or applicable UK laws. If you discover content in the Research Portal that you believe breaches copyright or violates any law, please contact openaccess@qub.ac.uk. 

adults- a feasibility study protocol

\section{Background}

Medicines are the most commonly used healthcare intervention in Aotearoa New Zealand (NZ). They are prescribed with the intent of providing therapeutic benefit i.e. to prevent, treat, or cure acute and chronic conditions. Medicines can, however, also cause harm, the risk of which increases with the number of medicines prescribed. ${ }^{1}$ Internationally, and in NZ, the number of medicines prescribed to an individual increases as people age. ${ }^{2}$ This occurs for a number of reasons including the increasing number of comorbidities with increasing age, and prescribing according to best practice guidelines for single disease states. ${ }^{3}$ As the number of medicines increases, so does the complexity of medicines regimens and potential for harm from medicines use in older adults. ${ }^{1}$ Adverse drug events (ADEs) are the cause of between 10 and $30 \%$ of older adult hospitalisations ${ }^{4}$, with an ADE prevalence rate of up to $35 \%$ in community-dwelling older adults. ${ }^{5}$ In older adults, potentially inappropriate prescribing (PIP) can result from the prescription of medicines with the known potential to cause more harm in older adults, and from failure to initiate medicines with the potential to treat or prevent medical conditions ${ }^{6}$ and are a major cause of ADEs (including morbidity, hospitalisation and mortality). ${ }^{7,8}$ There are a number of tools that can be used to measure PIP prevalence. ${ }^{9,10}$

Pharmacists have a role to play in improving medicine use and reducing medicine-related harm. Pharmacist-led medicines reviews have been shown to reduce ADEs and PIP in older adults. ${ }^{5,11}$ The term 'medicines review' is used widely, and can incorporate a number of activities (such as medicines education, adherence support and development of medicines management plans). For the purposes of this protocol, the term is used to describe an intentional, structured and critical review of medicines, carried out by health professionals, in discussion with the patient, and with the aim of agreeing on optimal medicines use to 
63

64

65

improve the quality, safety and appropriate use of medicines. ${ }^{12}$ Medicines reviews are particularly beneficial when the intervention is delivered by a pharmacist working as part of a collaborative, multi-disciplinary team, and, in the case of older adults, when the pharmacist has expertise in geriatric medicine. ${ }^{13} \mathrm{~A}$ recent Cochrane review showed that pharmacists based in the community (including community pharmacy, general practitioner (GP) practices and other primary care settings) may improve patient outcomes relating to measures which impact on the control of long-term conditions such as cardiovascular disease and diabetes. ${ }^{14}$

In NZ, inequities in health outcomes exist between Māori (Indigenous people of NZ) and non-Māori resulting from the unfair, differential allocation and distribution of resources and power related to the determinants of health. ${ }^{15}$ Māori experience worse health outcomes, including increased morbidity, increased avoidable mortality, ${ }^{16}$ and lower life expectancy, ${ }^{17}$ than non-Māori. These inequities occur across the spectrum of health services ${ }^{16,18-21}$ and include access to medicines and medicine-related services. ${ }^{21-24}$ Māori older adults are more likely to be prescribed 'high risk' medicines (those associated with an increased likelihood of harm) than non-Māori ${ }^{25}$ and are more likely to experience an adverse outcome resulting from the omission of an appropriate medicine, than the prescription of an inappropriate medicine. ${ }^{26}$ These inequities exist despite guarantees made under the Treaty of Waitangi, one of NZ's founding documents, which asserts the right of Māori to receive services and resources that reflect their needs as Māori, and which result in equitable outcomes. There is ever-growing evidence that the systems governing, legislating, administering and funding primary healthcare services fail to meet the needs of Māori. This has been formally recognised by the recent Waitangi Tribunal Hauora Report, ${ }^{27}$ as a breach of the Treaty of Waitangi. Service models which improve medicines use for Māori, including the access to medicines, and quality of prescribing are needed to support the attainment of health equity. ${ }^{28}$

\section{Study aim and objectives}


The aim of the current study is to test the feasibility of a pharmacist-led medicines review intervention, informed by Indigenous methodologies, for (and with) community-dwelling Māori older adults.

The study objectives are:

1. To test the acceptability of the medication review intervention.

2. To test the ability to recruit Māori older adults into the feasibility study.

3. To assess medicines knowledge, quality of life (QoL) and PIP scores in study population at baseline and post-intervention.

4. To report number of changes made to the medicines regimen as a result of the intervention.

5. To test the feasibility of the tools chosen to assess medicines knowledge, QoL and PIP, including administration time required.

6. To test the intervention practicality in relation to time required to deliver the intervention.

7. To assess whether modification to recruitment methods, intervention content or delivery, and methods of assessing outcomes is required prior to wider implementation and testing.

\section{Methods}

This research project will use a mixed methods approach to test the feasibility of a pharmacist-led medicines review intervention for community-dwelling Māori older adults. This study will be guided by kaupapa Māori research theory. ${ }^{29,30}$ 'Kaupapa Māori' is translated as a 'Māori way' or 'Māori ideology'31 and has developed as a research methodology over the last 4 decades. It aims to normalise Māori worldviews and ways of knowing, ${ }^{29}$ and take back space and power for Māori in the research process, ${ }^{32}$ which includes authentic Māori participation at all stages. Kaupapa Māori research affirms the right of Māori to actively participate in research. In the context of health this includes the right to partner with health providers to develop and receive relevant, appropriate and safe health services, and experience equitable health outcomes. It aims to have positive impact for Māori and comes from a place of dreaming of infinite possibilities for Māori. ${ }^{33}$ Kaupapa Māori methodology allows the incorporation of methods developed within different theoretical approaches, as long as they have relevance and can be utilised in a way that 
121 upholds the principles of kaupapa Māori theory and practice. In health service research this

122 means that tools developed within a biomedical framework may have relevance, and be

123 able to be applied, within a kaupapa Māori methodological approach.

124 Medicines review services, which include multiple practitioners as well as patients and

125 family, are complex interventions as there are multiple interacting components. ${ }^{34}$ This can

126 make the development, implementation and evaluation of an intervention more difficult,

127 hence the development of the United Kingdom Medical Research Council's guidance

128 document on the development of complex interventions. ${ }^{34}$ This document supports

129 kaupapa Māori approaches of authentically engaging with those receiving and delivering

130 interventions, and has been utilised to support the development of the proposed research.

131 The intervention to be tested in the current study has been informed through previous

132 research by the current authors (Phase $1 \mathrm{a}, 1 \mathrm{~b}$ and $2 \mathrm{a}$ - see Figure 1 ). In Phase 1a, in addition

133 to a systematic review, ${ }^{23}$ Māori older adults $(n=10)$ and health practitioners and commissioners of services $(n=11)$ were interviewed to inform the development of the intervention and outcomes measures (Figure 1). The proposed intervention and outcome measures were reported back to the interviewees with wider dissemination to community groups. The intervention was found to be acceptable within these groups.

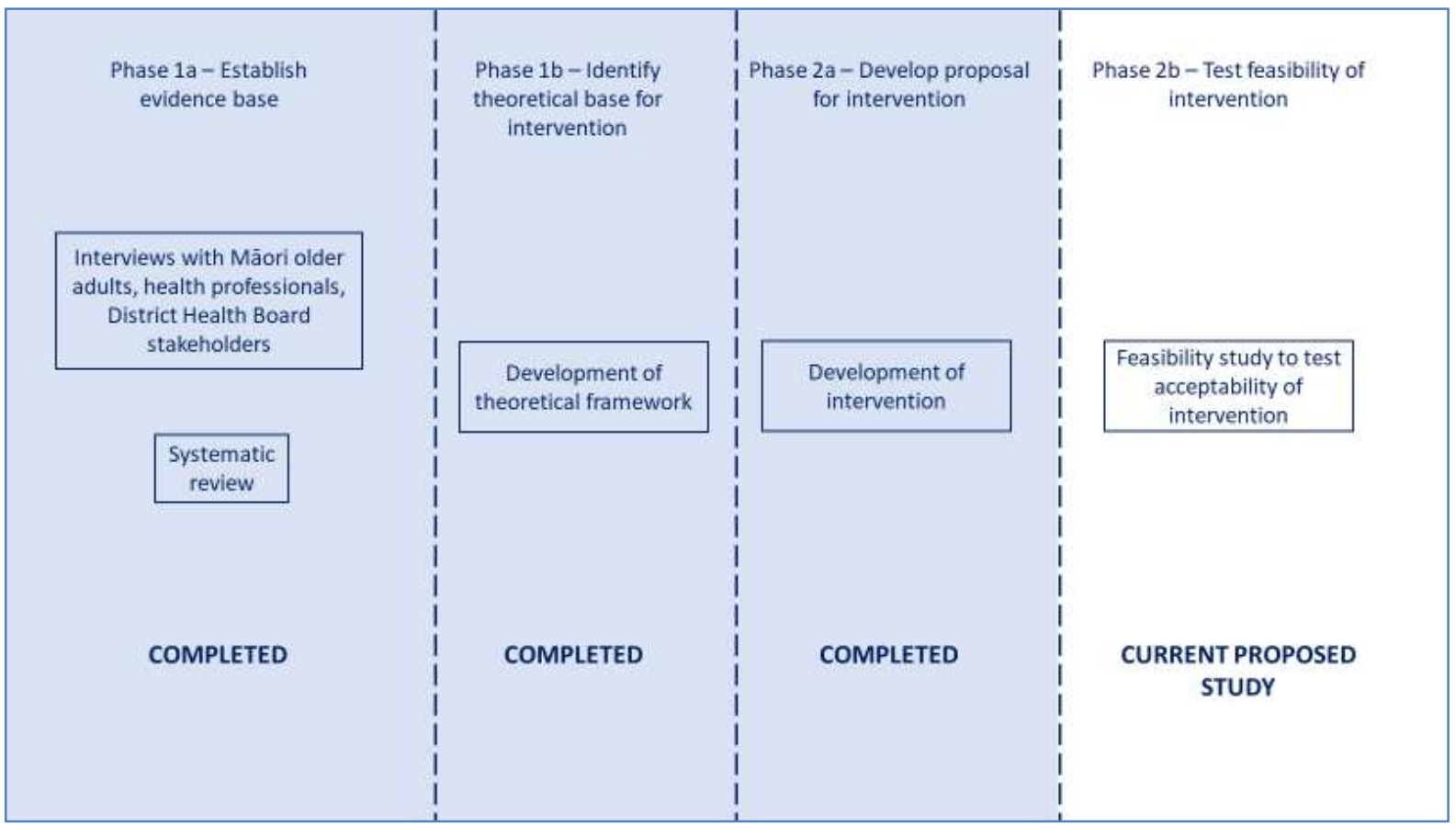


141 The intervention will be tested in a single-arm feasibility study with baseline and post-

142 intervention measurement of predefined outcomes. The study will be reported in

143 accordance with the CONSIDER statement ${ }^{35}$ aimed to strengthen the reporting of health research which involves Indigenous peoples to ultimately "advance Indigenous health outcomes", 35 and the CONSORT 2010 statement: extension to randomised pilot and

146 feasibility trials, ${ }^{36}$ as relevant to the non-randomised nature of the currently described 147 study.

Project oversight

An advisory team including Māori older adults, health professionals (including a Māori public health physician and health researcher) and the lead investigator (JH) will provide project oversight.

\section{Study population}

155 NZ is geographically divided into 20 District Health Boards (DHBs) which are responsible for the provision and funding of health services. Participants will be drawn from Waitematā District Health Board (WDHB), Auckland, NZ. Inclusion criteria for patients Māori ethnicity (self-identified) AND Community dwelling (not resident in rest home, private hospital or hospice) AND Taking 4 or more regular medicines for at least three months AND 55 years of age or older ( 55 years is often used as entry age for service funding and

168 
170 A number of recruitment strategies will be employed to meet the needs of Māori older adults, as informed by interviews with health professionals and Māori older adults in Phase

172 1a. Potential participants will be given a Participant Information Sheet and Brief Study

173 Outline via: GP practices (mail-out to all enrolled patients that meet eligibility criteria; during consultations; in waiting areas), community pharmacies, and during investigator (JH) presentations to Māori older adult groups. In addition, the Māori older adults involved in Phase 1a of this research, will be contacted directly and invited to participate (Māori older adult participants from Phase 1a wanted to have the option of being involved in service delivery developed as a result of the initial phases of this research. In kaupapa Māori methodology the research relationship continues past distinct research projects and requires long-term commitment and the ability for Māori to participate in ongoing translational work. Therefore, participants from Phase 1a will be given the option to participate in the subsequent phase investigated in this study). The information provided will outline the steps involved in the intervention, including baseline and post-intervention assessments. It will ask potential participants to contact the lead investigator to discuss participation. Those that find out about the project through 'word-of-mouth', and meet the inclusion criteria, will also be eligible to participate.

Setting and location of collection of eligibility information and consent Participants will be assessed for eligibility and consented by the lead investigator in a faceto-face meeting. The location of this meeting will be determined by the potential participant but may include GP practice, community centre or participant's home. At point of written informed consent, baseline demographic data will be collected. This will also include recording the recruitment method for the particular individual.

\section{Recruitment of general practices}

Where GP practices are used to support recruitment, convenience sampling will be used to recruit practices. Practices will be eligible if they are within WDHB. JH will approach GP practices, discuss the project and gain written consent from a staff member at the GP practice (for example clinical director, practice manager, GP, practice owner) with authority to give consent. 
203 Participant flow through the intervention and related outcome assessments is shown in

204 Figure 2.

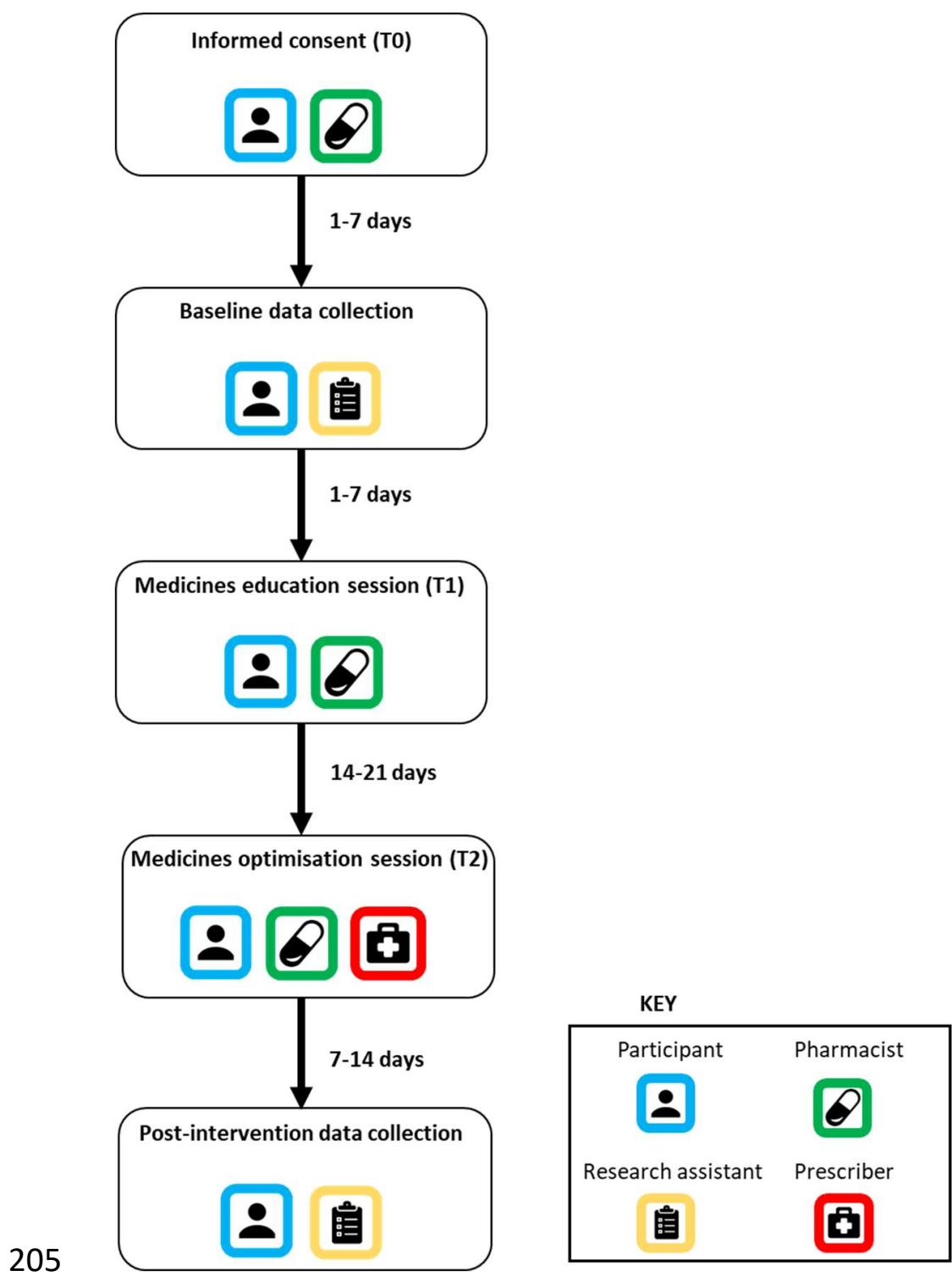

206 Figure 2. Process flow for participant for data collection and intervention

The intervention will be delivered by JH, a Māori clinical pharmacist with experience in older adult medicines reviews and who is leading all the phases of the service development.

The intervention will consist of 2 parts:

1. Medicines education session (participant and pharmacist) improve the participants' understanding of what the medicines are used for, 
potential side effects and likely length of treatment, as well as improving the pharmacist's understanding of what is important to the participant. Participants will be asked to bring their medicines along to the education session to guide discussions. Discussions will be guided by what the participant identifies as priority areas and will exert the participant's right for self-determination and control in their health journey. This component will include tasks to the level of Medicines Use Review (MUR) and Medicines Therapy Assessment (MTA) in the Pharmaceutical Society of NZ National Pharmacist Services Framework. ${ }^{37}$ These tasks include medicines reconciliation (including access to the electronic dispensed medicine repository Testsafe $\mathrm{e}^{\mathrm{TM}}$ by pharmacist prior to education session), discussion of medicines-related needs and goals of wellbeing, provision of relevant information resources, medicines effectiveness or ADEs, and discussion of medicines in the context of participant-identified goals of therapy.

\section{Medicines optimisation session (participant, pharmacist and prescriber)}

Prior to meeting with the participant and prescriber, the pharmacist will review clinical records via the WDHB electronic clinical portal (contains hospital discharge summaries, clinic letters, laboratory investigations, hospital inpatient observations, history of dispensed medicines). Following review, the prescriber, pharmacist and participant will meet and develop a medicines management plan. This session will align services described under MTA and Comprehensive Medicines Management (CMM) in the PSNZ Pharmacist Services Framework ${ }^{37}$ and the guidance set out in the Royal Pharmaceutical Society of Great Britain's Medicines Optimisation good practice guidance. ${ }^{38}$ The medicines optimisation session is optional with consent requested at point of initial consent. This session will occur 14-21 days after the medicines education session.

239 After each session, discussions will be summarised and sent to the participant's primary prescriber and community pharmacy either via post or secure electronic mail. This aspect of

241 the intervention allows for connected communication between those involved in provision of health care to the participant. Participants will also be able to invite family and/or support people to be present at these sessions. 
The medicines education component will take place in a location of the participant's choosing and may include their own home, GP practice or community centre. The medicines optimisation session will occur at the GP practice.

\section{Study Outcomes}

250

The primary outcome investigated in this feasibility study is the acceptability or otherwise of the intervention to participants, family members/support people and healthcare providers. The inclusion of these 3 groups has occurred in kaupapa Māori assessment previously ${ }^{39}$ and acknowledges that services can be evaluated from different view-points and experiences. Secondary outcomes: ability to recruit; medicines knowledge, PIP and QoL scores; number of medicine changes; resource required to deliver intervention and administer assessment tools (Table 1 ).

\section{Data Collection}

258 The assessment tools, methods of data collection and time points for data collection are detailed in Table 1. Participants will be assigned a random 4-digit study number to be used during data collection. Acceptability questionnaires have been developed specifically for this research project and are based on the theoretical framework developed in Phase $1 \mathrm{~b}$, in addition to review of other Indigenous outcomes measures and frameworks. ${ }^{40}$ Acceptability questions will ask participants to respond on a 5-point Likert scale with 'strongly agree' to 'strongly disagree' statements. Questions will focus on aspects relating to participant control and medicines knowledge, relevance and usefulness of the medicines review service, appropriateness of the pharmacist to deliver intervention, likelihood to use this type of service and refer others, and whether the care was culturally appropriate and delivered in a respectful and collaborative manner. In addition, there will be some openended questions relating to perceived value of the intervention and areas for improvement. All assessment tools which require correspondence with the participant for completion

271 (acceptability questionnaire, medicines knowledge and QoL) will be administered by a

272 research assistant to reduce potential bias due to participants having a therapeutic

273 relationship with the lead investigator. These will be administered via the telephone, with data collected using Qualtrics ${ }^{\mathrm{TM}}$ as the survey tool. 
Table 1. Study outcomes and tools, methods and time points for data collection.

\begin{tabular}{|c|c|c|c|c|}
\hline & Outcomes & Assessment tool & Method of data collection & $\begin{array}{l}\text { Time point of data } \\
\text { collection }\end{array}$ \\
\hline \multirow[t]{2}{*}{$\begin{array}{l}\text { Primary } \\
\text { outcome }\end{array}$} & \multirow[t]{2}{*}{ Acceptability } & Participant acceptability questionnaire & $\begin{array}{l}\text { Telephone administration by } \\
\text { research assistant; data } \\
\text { collection in Qualtrics }^{\mathrm{TM}}\end{array}$ & \multirow[t]{2}{*}{$\begin{array}{l}4 \text { weeks post- } \\
\text { intervention }\end{array}$} \\
\hline & & $\begin{array}{l}\text { Family member/support person acceptability } \\
\text { questionnaire }\end{array}$ & Online survey using Qualtrics ${ }^{\mathrm{TM}}$ & \\
\hline $\begin{array}{l}\text { Secondary } \\
\text { outcomes }\end{array}$ & Recruitment & $\begin{array}{l}\text { Recruitment methods detailed including time taken, cost } \\
\text { (e.g. postage). Approaches for participation by those not } \\
\text { eligible will also be noted and with the potential to } \\
\text { suggest changes to eligibility criteria for future studies. }\end{array}$ & Log book & Throughout study \\
\hline
\end{tabular}




\begin{tabular}{|c|c|c|c|}
\hline Participant QoL & Short Form (36) Health Survey (SF-36) & $\begin{array}{l}\text { Telephone administration by } \\
\text { research assistant; data } \\
\text { collection in Qualtrics }^{\mathrm{TM}}\end{array}$ & $\begin{array}{l}\text { Baseline and } 4 \\
\text { weeks post- } \\
\text { intervention }\end{array}$ \\
\hline & $\begin{array}{l}\text { Prescribers will be requested to estimate their time input } \\
\text { in relation to the intervention as part of the Prescriber } \\
\text { acceptability questionnaire. }\end{array}$ & Online survey using Qualtrics ${ }^{\mathrm{TM}}$ & $\begin{array}{l}\text { Post-intervention } \\
\text { for all patient } \\
\text { participants }\end{array}$ \\
\hline
\end{tabular}


278 Although sample size justification is important, sample size calculations may not be appropriate in feasibility studies. A variety of methods for justification of sample size have been utilised in feasibility studies. ${ }^{42}$ Most of these are 'rule of thumb' methods which are regarded as 'flat' methods as they do not account for the potential size of the main trial. ${ }^{46}$ Using the 'rule of thumb' methods, recommended sample sizes range from $12-70 .{ }^{46} \mathrm{~A}$ recent paper suggests that if rule of thumb methods are to be used, they should at least vary according to standardised difference in outcome measures; smaller standardised differences require a larger sample size and vice versa. In this study, the SF- $36^{41}$ serves as a basis for sample size justification as the SF-36 has been widely validated across a range of clinical contexts including pharmaceutical care services. ${ }^{43} \mathrm{~A}$ meta-analysis of the effect of pharmaceutical care interventions on SF- $36^{43}$ showed a standardised mean difference of 0.39 for general health. Theoretical modelling of sample size calculation has been performed for pilot randomised trials. ${ }^{44}$ Based on the standardised mean difference of 0.39 , if a randomised pilot were to be undertaken using this approach, each treatment arm for a $90 \%$ powered trial should have 21 or 33 participants for a $80 \%$ or $95 \%$ upper confidence level respectively. ${ }^{44}$ Although this feasibility study is not randomised or controlled, these figures can be used as an estimation in our work. We aim to recruit and deliver the intervention to 30 participants. Results from this feasibility study will be used to guide sample size calculations in future studies. ${ }^{45}$

Analytical Methods

Data analysis will consist of quantitative and qualitative methods. Simple descriptive statistics will be used to summarise the data relating to acceptability questionnaire agree-disagree statements, recruitment methods, medicines knowledge, QoL, PIP and time resource required to deliver intervention and apply assessment tools. Qualitative data analysis using a general inductive approach within a critical kaupapa Māori theoretical framework will be used to assess open-ended questions in acceptability questionnaires, to describe appropriateness of assessment tools and report on changes to the intervention and/or research processes that should be revised or changes prior to wider testing/implementation. Due to the small sample size, subgroup analysis will not be performed. Quantitative data analysis will be conducted 
310 Although internationally, pharmacist-led medicines review services have been shown to

311 reduce inappropriate prescribing and adverse drug events in the older adult population, ${ }^{5,11}$

312 their ability to respond to the needs of Māori older adults, and the contribution they may

313 make to achieving health equity in NZ, remains unknown. ${ }^{23}$ Māori older adults report that

314 medicines are a key factor contributing to their wellness ${ }^{46}$ and there is a need to develop

315 culturally congruent medicines-related services to support this for Māori. ${ }^{28}$ The intervention

316 to be tested in this study includes pharmacist-led medicines education and optimisation

317 components, was designed using kaupapa Māori informed methods and could shape further

318 health service development in this space.

319 The intervention to be tested was informed by Māori older adults' desire to be more in

320 control of their medicines management journey. Therefore, the medicines optimisation

321 component was optional to allow participants choice over whether the research pharmacist

322 had full access to their clinical notes and whether they were present in a meeting with their

323 prescriber. The intervention also includes participant, pharmacist and prescriber at the point

324 where decisions are made about medicines management.

\section{$325 \quad$ Limitations}

326 A small sample size and a short follow-up period will be used which is appropriate for a

327 feasibility study ${ }^{47}$ to reduce the risk of committing extensive resource when intervention

328 content and delivery, and outcomes evaluation, may need modification. Clinical outcomes,

329 such as mortality and hospitalisation, are unlikely to be significantly impacted during the

330 study period given the type of intervention and small sample size. Given the potential for

331 diversity of co-morbidity and medicines utilisation in this study (which is not focused on a

332 single disease state or medicine) it is unlikely that there will be a significant impact on

333 particular participant clinical observations (such as blood pressure). Therefore, this study

334 does not seek to report on these or use them as outcome measures. The approach of

335 focusing on participant and prescriber acceptability was supported by health practitioner

336 and DHB stakeholder interviews undertaken in Phase 1b. 
339 Due to the small participant numbers, intervention implementation and results are unlikely

340 to be generalisable, especially in populations outside Waitematā DHB. The study will be

341 conducted in participants who either reside or access primary healthcare services in

342 Waitematā DHB, the largest DHB in NZ, with a predominately urban population and with the 343 highest life expectancy.

344 A complex intervention is being studied in this research. ${ }^{34}$ Multiple practitioners and

345 processes are involved, and thus being able to replicate the results of this study using

346 different practitioners, in different settings, is unlikely. However, it is intended that this

347 study will generate insights into which elements of the intervention were important (or not)

348 and the ability to administer evaluation tools. The intervention and assessment tools can

349 then be adapted, as needed, prior to the intervention being tested in a larger study. This

350 pragmatic approach is reflective of real-life interventions and learnings from this may be

351 relevant to other research settings, including other feasibility studies relating to health

352 service development.

353

354 This study serves as an example of health service design, delivery and evaluation, informed by Indigenous knowledge and methodology, developed explicitly to address inequities in health outcomes for, and with, Māori.

357

Ethics and dissemination

Ethical approval was granted by the Northern B Health and Disability Committee (19/NTB/106). The study has been registered with the Australia and New Zealand Clinical Trials Registry (ACTRN12619001070123) and the full trial protocol is publicly available on this site.

363 A dissemination plan will be developed by the research team in collaboration with the project advisory group. Results, including suggested refinements to intervention and study design, will be disseminated to various groups and stakeholders within WDHB and nationally including Māori communities, Māori older adult groups, health practitioners, DHB funders and planners, GP practices, and pharmacy and primary care sector groups. Results will be reported as research articles in international peer-reviewed journals, at international and national conferences and in the lead author's doctoral thesis. 
None

\section{$374 \quad$ Funding}

375 This work was supported by the Health Research Council of NZ [Clinical Research Training

376 Fellowship; HRC Ref: 17/137] and a Waitematā DHB Small Contestable Grant [Hikaka/2017].

377 Funders had no role or influence over study design; the collection, analysis and

378 interpretation of data; in the writing of the report; or the decision to submit the article for 379 publication.

\section{References}

381 1. Fulton MM, Riley Allen E. Polypharmacy in the elderly: a literature review. Journal of the American Association of Nurse Practitioners. 2005;17(4):123-132.

383

2. Health Quality and Safety Committee. Polypharmacy in people aged 65 and over [Internet]. 2019 [cited 2019 Mar 15]. Available from: https://www.hqsc.govt.nz/ourprogrammes/health-quality-evaluation/projects/atlas-of-healthcarevariation/polypharmacy/

3. Johansson T, Abuzahra ME, Keller S, Mann E, Faller B, Sommerauer C, et al. Impact of strategies to reduce polypharmacy on clinically relevant endpoints: a systematic review and meta-analysis. British Journal of Clinical Pharmacology. 2016;82(2):532-48.

4. Parameswaran Nair, Chalmers L, Peterson G, Bereznicki B, Castelino R, Bereznicki L. Hospitalization in older patients due to adverse drug reactions - the need for a prediction tool. Clinical Interventions in Ageing. 2016;11:497-505.

5. Gray SL, Hart LA, Perera S, Semla TP, Schmader KE, Hanlon JT. Meta-analysis of Interventions to Reduce Adverse Drug Reactions in Older Adults. Journal of the American Geriatrics Society. 2018;66(2):282-288.

6. Rankin A, Cadogan CA, Patterson SM, Kerse N, Cardwell CR, Bradley MC, et al. Interventions to improve the appropriate use of polypharmacy for older people. Cochrane Database of Systematic Reviews. 2018;(19). 
7. Hamilton H, Gallagher P, Ryan C, Byrne S, O'Mahony D. Potentially inappropriate medications defined by STOPP criteria and the risk of adverse drug events in older hospitalized patients. Archives of internal medicine. 2011;171(11):1013-1019.

8. Klarin I, Wimo A, Fastbom J. The association of inappropriate drug use with hospitalisation and mortality. Drugs \& aging. 2005;22(1):69-82.

9. O'Mahony D, O'Sullivan D, Byrne S, O'Connor MN, Ryan C, Gallagher P. STOPP/START criteria for potentially inappropriate prescribing in older people: version 2. Age Ageing. 2015 Mar 1;44(2):213-8.

10. American Geriatrics Society Beers Criteria Update Expert Panel. American Geriatrics Society 2019 Updated AGS Beers Criteria ${ }^{\circledR}$ for Potentially Inappropriate Medication Use in Older Adults. Journal of the American Geriatrics Society. 2019;67(4):674-94.

11. Riordan DO, Walsh KA, Galvin R, Sinnott C, Kearney PM, Byrne S. The effect of pharmacist-led interventions in optimising prescribing in older adults in primary care: a systematic review. SAGE open medicine. 2016;4:1-18.

12. Blenkinsopp A, Bond C, Raynor DK. Medication reviews. British Journal of Clinical Pharmacology. 2012;74(4):573-80.

13. Spinewine $A$, Fialová $D$, Byrne $S$. The role of the pharmacist in optimizing pharmacotherapy in older people. Drugs \& aging. 2012;29(6):495-510.

14. de Barra $M$, Scott $C$, Scott $N$, Johnston $M$, de Bruin $M$, Nkansah $N$, et al. Pharmacist services for non-hospitalised patients. Cochrane Database of Systematic Reviews. 2018;

15. Robson B. Economic Determinants of Māori Health and Disparities: a review for Te Rōpū Tohutohu i te Hauora Tūmatanui (Public Health Advisory Committee of the National Health Committee). Wellington: Te Ropu Rangahau Hauora a Eru Pomare, University of Otago; 2004. 
16. Walsh M, Grey C. The contribution of avoidable mortality to the life expectancy gap in Māori and Pacific populations in New Zealand-a decomposition analysis. The New Zealand Medical Journal. 2019;132(1492).

17. Ministry of Health. Tatau Kahakura: Māori Health Chart Book 2015. 3rd ed. Wellington: Ministry of Health; 2015.

18. Crengle S, Lay-Yee, R, Davis, P, Pearson, J. A comparison of Māori and non-Māori patient visits to doctors: The National Primary Medical Care Survey (NatMedCa) :2001/02. Wellington, N.Z.: Ministry of Health; 2005.

19. Curtis E, Harwood M, Riddell T, Robson B, Harris R, Mills C, et al. Access and Society as Determinants of Ischaemic Heart Disease in Indigenous Populations. Heart, Lung and Circulation. 2010 May;19(5-6):316-24.

20. Rahiri J-L, Lauti M, Harwood M, MacCormick AD, Hill AG. Ethnic disparities in rates of publicly funded bariatric surgery in New Zealand (2009-2014). ANZ Journal of Surgery. 2018 May 1;88(5):E366-9.

21. Health Quality \& Safety Commission. A window on the quality of Aotearoa New Zealand's health care 2019. Wellington: Health Quality \& Safety Commission; 2019.

22. Metcalfe S, Beyene K, Urlich J, Jones R, proffitt C, Harrison J, et al. Te Wero tonu-the challenge continues: Māori access to medicines 2006/07-2012/13 update. New Zealand Medical Journal. 2018;131(1485).

23. Hikaka J, Hughes C, Jones R, Connolly MJ, Martini N. A systematic review of pharmacist-led medicines review services in New Zealand - is there equity for Māori older adults? Research in Social and Administrative Pharmacy [Internet]. 2019 Jan 14 [cited 2019 Feb 8]; Available from:

http://www.sciencedirect.com/science/article/pii/S1551741118308477

24. Jatrana $S$, Crampton $P$, Norris P. Ethnic differences in access to prescription medication because of cost in New Zealand. Journal of Epidemiology \& Community Health. 2011 May 1;65(5):454-60. 
25. Nishtala PS, Narayan SW, Wang T, Hilmer SN. Associations of drug burden index with falls, general practitioner visits, and mortality in older people. Pharmacoepidemiology and drug safety. 2014;23(7):753-758.

26. Ryan C, Teh R, Moyes S, Wilkinson T, Connolly MJ, Rolleston A, et al. Quality of prescribing predicts hospitalisation in octogenarians: LiLACS NZ. BMC Geriatrics (under review). 2019;

27. Waitangi Tribunal. Hauora -Report on Stage One of the Health Services and Outcomes Kaupapa Inquiry. Wai 2575. Wellington: Waitangi Tribunal; 2019. Report No.: Prepublication version.

28. Te Karu L, Bryant L, Harwood M, Arroll B. Achieving health equity in Aotearoa New Zealand: the contribution of medicines optimisation. Journal of Primary Health Care. 2018;10(1):5.

29. Pihama L. Kaupapa Māori theory: transforming theory in Aotearoa. He Pukenga Korero. 2010;9(2):5-14.

30. Smith G. Kaupapa Maori Theory: Theorizing Indigenous Transformation of Education and Schooling. In: Kaupapa Māori symposium. Auckland, N.Z.; 2003.

31. Brewer KM, Harwood MLN, McCann CM, Crengle SM, Worrall LE. The Use of Interpretive Description Within Kaupapa Māori Research. Qual Health Res. 2014 Sep 1;24(9):1287-97.

32. Smith LT. Decolonising methodologies: Research and indigenous peoples. 2nd ed. London: Zed Books; 2012.

33. Curtis E. Indigenous positioning in health research: The importance of Kaupapa Māori theory-informed practice. AlterNative: An International Journal of Indigenous Peoples. 2016;12(4):396-410.

34. Craig P, Dieppe P, Macintyre S, Michie S, Nazareth I, Petticrew M, et al. Developing and evaluating complex interventions: the new Medical Research Council guidance. BMJ. 2008;337:a1655.

\begin{tabular}{|l|l|l|}
\hline A pharmacist-led medicines review intervention in community-dwelling Mãori older adults- a feasibility study & Apr 2019 & $\begin{array}{l}\text { Page } 18 \text { of } \\
\mathbf{2 1}\end{array}$ \\
\hline
\end{tabular}


35. Huria T, Palmer S, Pitama S, Beckert L, Lacey C, Ewen S, et al. Consolidated criteria for strengthening reporting of health research involving indigenous peoples: the CONSIDER statement. BMC Medical Research Methodology [Internet]. 2019 [cited 2019 Aug 13];19(173). Available from: https://www.readcube.com/articles/10.1186/s12874-019-0815-8

36. Eldridge SM, Chan CL, Campbell MJ, Bond CM, Hopewell S, Thabane L, et al. CONSORT 2010 statement: extension to randomised pilot and feasibility trials. Pilot and Feasibility Studies. 2016 Dec;2(1):64.

37. Pharmaceutical Society of New Zealand. New Zealand National Pharmacist Services Framework 2014. 2014.

38. Picton $\mathrm{C}$, Wright $\mathrm{H}$. Medicines optimisation: Helping patients to make the most of medicines. Good practice guidance for healthcare professionals in England. Royal Pharmaceutical Society; 2013.

39. Kingi TK, Durie M. "Hua Oranga” A Maori Measure of Mental Health Outcome. Palmerston North: Massey University; 1999 p. 79.

40. Durie M. Te Pou Matakana - Outcomes framework [Internet]. Te Pou Matakana Commissioning Agency; 2014. Available from: https://www.tepoumatakana.com/wpcontent/uploads/2017/03/Te-Pou-Matakana-Outcomes-Framework.pdf

41. Ware JE, Kosinski M. SF-36 Physical and Mental Health Summary Scales: A Manual for Users of Version 1. 2nd ed. Lincoln, RI: Quality Metric, Inc; 2001.

42. Billingham SA, Whitehead AL, Julious SA. An audit of sample sizes for pilot and feasibility trials being undertaken in the United Kingdom registered in the United Kingdom Clinical Research Network database. BMC Med Res Methodol. 2013 Aug 20;13:104.

43. Mohammed MA, Moles RJ, Chen TF. Impact of Pharmaceutical Care Interventions on Health-Related Quality-of-Life Outcomes: A Systematic Review and Meta-analysis. Ann Pharmacother. 2016 Oct 1;50(10):862-81.

\begin{tabular}{|l|l|l|}
\hline A pharmacist-led medicines review intervention in community-dwelling Māori older adults- a feasibility study & Apr 2019 & $\begin{array}{l}\text { Page } 19 \text { of } \\
\mathbf{2 1}\end{array}$ \\
\hline
\end{tabular}


505

506

507

508

509

510

511

512

513

514

515

516

517

518

519

520

521

44. Whitehead AL, Julious SA, Cooper CL, Campbell MJ. Estimating the sample size for a pilot randomised trial to minimise the overall trial sample size for the external pilot and main trial for a continuous outcome variable. Statistical Methods in Medical Research. 2016 Jun;25(3):1057-73.

45. Eldridge SM, Lancaster GA, Campbell MJ, Thabane L, Hopewell S, Coleman CL, et al. Defining Feasibility and Pilot Studies in Preparation for Randomised Controlled Trials: Development of a Conceptual Framework. PLoS One [Internet]. 2016 Mar 15 [cited 2019 Apr 29];11(3). Available from:

https://www.ncbi.nlm.nih.gov/pmc/articles/PMC4792418/

46. Allport T, Martin G, White H. Kaumātuatanga - The needs and wellbeing of older Māori [Internet]. Auckland, N.Z.: Te Pou Matakana; 2018. Available from: https://www.tepoumatakana.com/wp-content/uploads/2018/10/7A.Kaum\%C4\%81tuatanga-The-Needs-and-Wellbeing-of-Older-M\%C4\%81ori.pdf

47. Bowen DJ, Kreuter M, Spring B, Cofta-Woerpel L, Linnan L, Weiner D, et al. How We Design Feasibility Studies. American Journal of Preventive Medicine. 2009 May;36(5):452-7. 
